\title{
Recurrent stroke in varicella zoster associated
}

\section{vasculopathy}

\author{
Pradeep Kumar Maurya $D M^{1}$ iD, Ajai Kumar Singh $D M^{2}$ iD, Ashutosh Tiwari $D M^{3}$ iD, \\ Abdul Qavi $\boldsymbol{D M}^{4}$ iD \\ 1,2,4 Department of Neurology, Dr. Ram Manohar Lohia Institute of Medical Sciences, Lucknow, Uttar Pradesh, India \\ ${ }^{3}$ Department of Neurology, All India Institute of Medical Sciences, Rishikesh, Uttarakhand, India
}

\begin{abstract}
Varicella zoster virus (VZV) is well known for its neurotropism, primary infection and reactivation after variable latency periods. After reactivation from spinal or cranial nerve ganglia, viruses can affect the central nervous system and cranial vasculature via transaxonal migration followed by transmural spread from the adventitial layer to the intima. Stroke can occur following primary infection by VZV (varicella) or after reactivation (zoster). These infectious vasculitides by VZV can lead to unifocal or multifocal ischemic and hemorrhagic stroke either after cranial nerve or spinal dermatomal zoster. Usual difference between immunocompetent versus immunocompromised individuals is involvement of unifocal large vessel vasculopathy in the former while multifocal small vessel in later. This vasculopathy in some cases may be progressive leading to recurrent stroke even after antiviral treatment. Diagnosis becomes challenging and needs a high degree of suspicion in immunocompetent, younger individuals, in absence of rash and when there are comorbidities. We report a case of elderly immunocompetent women, who developed multifocal infarcts followed by ventricular and subarachnoid haemorrhage after thoracic varicella zoster. Diagnosis was confirmed by the presence of anti-VZV IgG antibodies in the cerebrospinal fluid. In view of the diverse clinic-radiological spectrum of VZV vasculopathy, early recognition of this clinical entity is warranted for improved outcome.
\end{abstract}

Key words: Intracerebral bleed, Stroke, Subarachnoid haemorrhage, Varicella zoster, Vasculopathy.

\section{Introduction}

$\mathrm{T}$ he varicella zoster virus (VZV) is a neurotropic DNA virus and after primary infection, it remains inactive in the neural ganglia of cranial or spinal nerves. Due to altered host immunity the reactivation can occur after

Access this article online
Website: https://www.nepjol.info/index.php/NJN
DOI: https://doi.org/10.3126/njn.v18i3.34994
HOW TO CITE
Maurya PK, Singh AK, Tiwari A, Qavi A. Recurrent stroke in
varicella zoster associated with vasculopathy. NJNS. 2021;18(3):61-
4.

Address for correspondence:

Dr. Pradeep Kumar Maurya

Department of Neurology,

Dr. Ram Manohar Lohia Institute of Medical Sciences,

Lucknow, Uttar Pradesh, India.

E-mail: pkm730@gmail.com

Phone: +91-9839129968

Copyright (C 2021 Nepalese Society of Neurosurgeons (NESON)

ISSN: 1813-1948 (Print), 1813-1956 (Online)

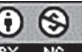

This work is licensed under a Creative Commons Attribution-Non Commercial 4.0 International License. a variable latency period and the virus again affects the nervous system and cranial vasculature. These infectious vasculitides of VZV can lead to ischemic and hemorrhagic stroke of various types with variable course which depends on host immune status. ${ }^{1}$ Though not a characteristic feature; the vasculopathy reported to be unifocal large vessel in immunocompetent and multifocal small vessel in immunocompromised patients. ${ }^{1}$ Vasculopathy can be an isolated event or may be recurrent due to progressive vasculopathy. ${ }^{2,3}$ We report a case of immunocompetent women, who presented with multifocal ischemic stroke followed by ventricular and subarachnoid haemorrhage due to progressive vasculopathy after thoracic varicella zoster. Diagnosis was confirmed by positive anti VZV IgG antibody in cerebrospinal fluid and excluding other causes. After administration of antiviral treatment, her clinical course was stabilised.

\section{Case report}

A 55-year-old lady with hypertension developed sudden onset right hemiparesis with speech disturbance one month back. She was admitted at a local hospital and magnetic resonance imaging (MRI) of the brain revealed acute infarct in the left basal ganglia, bilateral internal capsule and cerebellar vermis (Figure 1a). MR 


\section{Maurya et al}

angiography of intracranial vessels was unremarkable. She was improving on antiplatelets, antihypertensive and supportive treatment. One month after the initial stroke she again developed sudden-onset altered sensorium with respiratory distress and was admitted to our institute. She was put on a mechanical ventilator. Neurological examination revealed the Glasgow Coma Scale (GCS) of 9/15 (E4M4V1) and right upper motor neuron facial palsy. Motor examination showed right hemiplegia and ipsilateral plantars extensor. Old healed zoster rash over left thoracic region was noted (Figure 1b). History of vesicular lesion over the same region 2 months back was confirmed by relatives. Her non-contrast computed tomography (CT) brain revealed subarachnoid haemorrhage in the left sylvian fissure, interpeduncular cistern and ventricles (Figure 1c). CT angiography of the brain revealed a small aneurysm in the distal branches of the right middle cerebral artery (Figure 1d). She was detected to be diabetic and managed with oral hypoglycemic agents. In view of multifocal infarct in anterior and posterior circulation followed by subarachnoid and ventricular bleed with past history of zoster, the possibility of vasculopathy due to VZV was kept with the aim to rule out other etiologies. Her haemogram, renal, liver function test and thyroid profile were normal. Her serum antibody for human immunodeficiency virus, hepatitis $\mathrm{B}$ and $\mathrm{C}$ were negative. Her echocardiography and carotid doppler were normal. Chest X-ray and ultrasonography of the abdomen were unremarkable. Her ESR was elevated (101), c reactive protein was positive. Her serum for rheumatoid factor, antinuclear antibody and rapid plasma reagin (RPR) for syphilis were negative. Her cerebrospinal fluid (CSF) analysis showed mildly elevated proteins $(105 \mathrm{mg} / \mathrm{dL})$ with normal sugar $(74 \mathrm{mg} / \mathrm{dL}), 5$ mononuclear cells, without red blood cells. The CSF was also negative for bacteria, tuberculosis and cryptococcal infection. Her CSF came positive for anti-VZV IgG antibody; cut-off index (COI=2.19; $<0.8$ Negative and $>1.2$ Positive) while IgM was negative suggesting intrathecal synthesis of antibody. The antibody titres were not available. Her CSF polymerase chain reaction for VZV and herpes simplex virus -1 were negative. She was treated with valacyclovir (3gm/day in three divided doses) for 14 days. She was discharged after $2 \& 1 / 2$ month of hospitalization in stable condition. Her modified Rankin score was 3 at 6 month follow up. Antiviral treatment resulted in stabilization of disease. Repeat CT angiography of the brain performed at 6 month showed persistence of aneurysm.

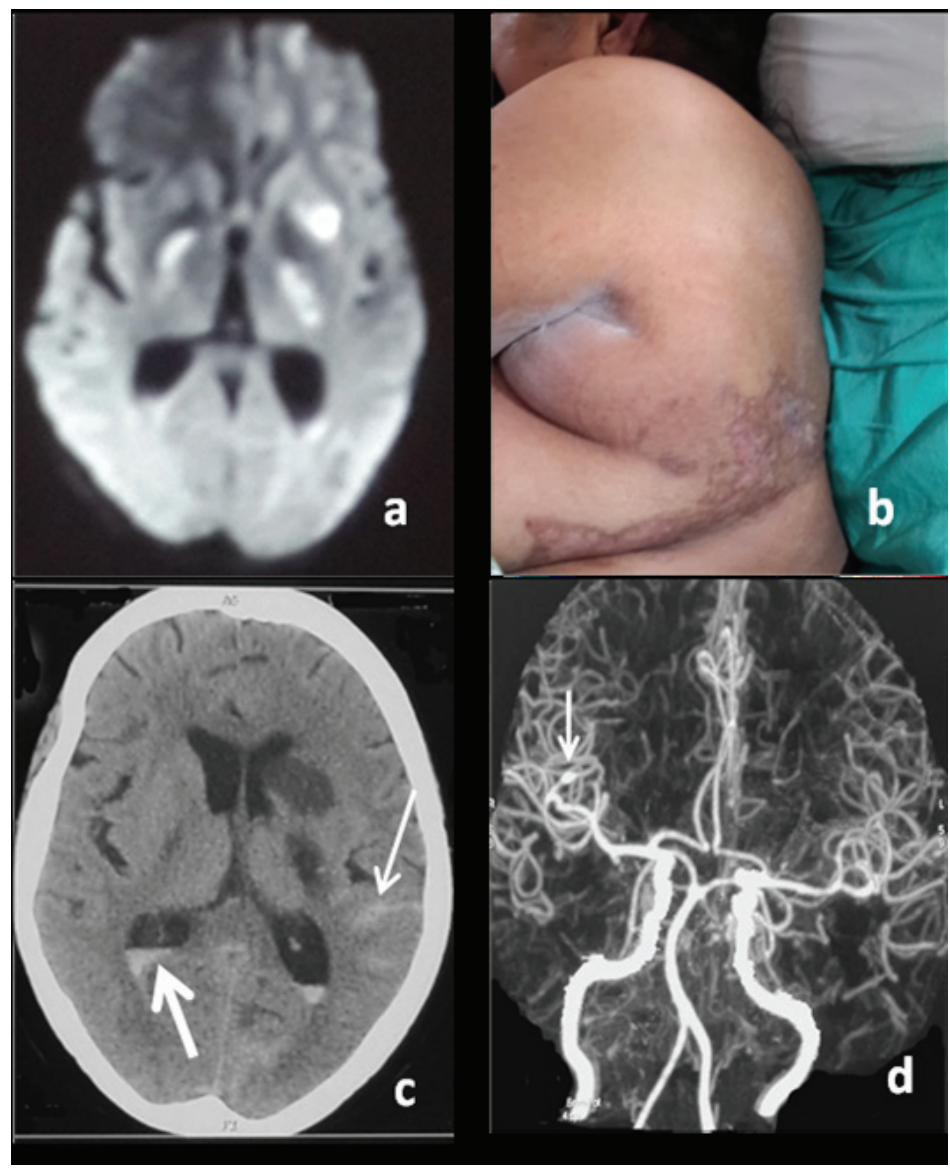

Figure 1(a): Diffusion-weighted magnetic resonance imaging of brain showing acute infarct in left putamen and bilateral internal capsule.

1(b): Old zoster rash over left thoracic dermatome.

1(c): Computed Tomography (CT) brain showing subarachnoid haemorrhage (thin arrow), intraventricular bleed (thick arrow) and old infarcts.

1(d): CT angiography brain showing distal right middle cerebral artery aneurysm (arrow). 


\section{Recurrent stroke associated with VZV vasculopathy}

\section{Discussion}

There has been an increased recognition, reporting and widened clinical spectrum of VZV vasculopathy in recent years. The pathogenesis of vascular remodelling is yet unclear. Proposed pathogenic mechanism is transaxonal migration of VZV form neural ganglia to cerebral arteries and resultant vasculopathy can easily explains the ipsilateral zoster ophthalmicus with contralateral hemiplegia, a classic presentation reported in literature. ${ }^{1}$ The evidence came forth form three VZV proven vasculopathy patients, when immunohistochemical analysis was performed on cerebral and temporal arteries revealed reactivated VZV antigen early in outer adventitial layer followed by media and intima during progression of disease. ${ }^{4}$ However cerebral VZV vasculopathy have been documented in sacral and thoracic dermatomal zoster as well. ${ }^{5.6}$ As in our case the vasculopathy at topographically distant site form preceding zoster rash may be due to simultaneous reactivation of virus in spinal and cranial ganglia (without rash) is likely possibility as this hypothesis has been suggested in the literature. ${ }^{5}$ Diverse and nonspecific clinical and neuroimaging features makes the diagnosis challenging. All of these complications are recognized to be due to vasculitis affecting small and/or large vessels due to direct infection of the vessel wall and vasculitis causing thrombosis. Other causes of infectious and non-infectious vasculitis to be investigated in appropriate clinical settings. Giant cell arteritis should be considered in elderly, which may have clinical features like headache, scalp tenderness and vision loss along with raised inflammatory markers like ESR and C-reactive protein in absence of rash; needs therapy with glucocorticoids. Although ESR was elevated and c-reactive protein was positive in our case, she did not receive steroids and improved with antiviral treatment. ANA was negative in our case, which is considered as the primary screening test for autoimmune disease. The arterial biopsy may demonstrate the presence of VZV antigen but it was not performed in our case as she survived and improved. Disruption of the internal elastic lamina in cerebral arteries infected with VZV can result in a weakened vessel wall that leads to dolichoectasia or aneurysm with subarachnoid or intracerebral haemorrhage. ${ }^{7,8}$ Progressive VZV vasculopathy resulting in recurrent stroke is well reported in literature. A 69 -year-old immunocompromised female developed 3 episodes of ischemic infarct in same arterial territory with a preceding history of zoster ophthalmicus. ${ }^{9}$ Diagnosis was confirmed by demonstration of anti VZV IgG antibody and positive PCR in CSF. Black blood MRI sequence was used to document the vasculopathy. In another case a 51-year-old female with a history of scleroderma had stepwise progression of focal neurological deficit over the duration of three month despite acyclovir therapy. In the absence of rash, diagnosis was established by VZV antibody in CSF. Repeat course of acyclovir along with steroid resulted in a stabilised course without significant clinical improvement. ${ }^{2}$ Our patient developed recurrent stroke (ischemic and hemorrhagic) after thoracic zoster and her CSF anti IgG for VZV came positive. Liberman $\mathrm{AL}$ et al reported a 41-year-old lady with systemic lupus erythematosus on immunosuppressant developed headache followed by zoster over multiple dermatomes. Her CSF was inflammatory and CT brain revealed SAH at multiple locations. ${ }^{7}$ PCR revealed VZV deoxyribonucleic acid in CSF. She was treated with intravenous acyclovir for 21 days but two months later her headache worsened. Repeat CT brain revealed SAH at multiple locations and digital subtraction angiography (DSA) revealed 9 aneurysms. A repeat course of acyclovir and steroid resulted in the resolution of a few aneurysms. Pathogenic mechanism for development of aneurysm was supposed to be intense inflammatory response which led to damage of vessel wall. ${ }^{7}$ As only CT angiography was performed and repeated in our case which revealed persistence of single aneurysm, whereas DSA can detect smaller aneurysm but could not be done in our case. Takeshita J et al reported simultaneous ischemic and hemorrhagic stroke in a patient with zoster rash over buttocks and it was consistent with VZV vasculopathy; diagnosis was made by positive zoster DNA in CSF. ${ }^{10}$ Cerebellar haemorrhage due to VZV vasculitis following zoster has also been reported. ${ }^{11}$ As entry of virus to anterior circulation can be easily explained by spread from trigeminal ganglia, whereas affliction of posterior circulation arteries lies on other proposed mechanism of viral spread from other pathways. ${ }^{11}$ This clinical entity may constitute a diagnostic challenge to clinicians as stroke may occur weeks or months after stroke, absence of rash and CSF pleocytosis in $1 / 3^{\text {rd }}$ of patients. ${ }^{12}$ The sensitivity of VZV PCR and anti VZV IgG in CSF are reported to be $30 \%$ and $93 \%$ respectively. ${ }^{13}$ The determination of anti VZV antibody in the CSF amid the course of vasculopathy strongly indicate that the incident stroke is related to concurrent VZV infection. It is further supported by the fact that the production and circulation of CSF is 3 times of total volume present in normal adults. ${ }^{5}$ The VZV antibody would be undetectable unless the infectious vasculopathy has a protracted course. The presence of antibody against VZV is considered diagnostic even though the PCR for VZV DNA is not identified as it is detectable early in the course. ${ }^{13}$ Although the detection of VZV DNA by PCR and IgG antibody against VZV are both considered diagnostic but negative PCR does not exclude the diagnosis; the only negative result for both can firmly exclude the possibility of VZV vasculopathy. ${ }^{13}$ As in our patient the IgG antibody against VZV was detected 2 month after the zoster rash suggesting active vasculopathy. Due to 
productive infection of VZV, treatment with acyclovir/ valacyclovir is justified and should be promptly instituted. Optimal therapeutic regimen is yet to be determined in the absence of controlled clinical trials. However intravenous acyclovir for 14 days along with short course of prednisone (5 days) have been advocated by some authors based on clinical experience. ${ }^{13}$ In selected cases higher doses of acyclovir (15 mg/kg q8h) and extended duration (21 days) and repeat course of treatment have been used., ${ }^{2,14}$

Our case highlights that diagnosing VZV vasculopathy in elderly may be difficult in the presence of other comorbid risk factors. This rare clinical entity should be considered even in dermatomal zoster. The awareness regarding the protean manifestations of VZV vasculopathy can lead to early diagnosis and treatment.

\section{Conflict of interest: None}

Financial support: Nil

\section{References}

1. Gilden D, Cohrs RJ, Mahalingam R, Nagel MA. Varicella zoster virus vasculopathies: Diverse clinical manifestations, laboratory features, pathogenesis, and treatment. Lancet Neurol. 2009;8(8):731-40. https://doi.org/10.1016/S1474-4422(09)70134-6

2. Russman AN, Lederman RJ, Calabrese LH, Embi PJ, Forghani B, Gilden DH. Multifocal varicellazoster virus vasculopathy without rash. Arch Neurol. 2003;60(11):1607-9. https://doi.org/10.1001/ archneur.60.11.1607

3. West SL, Newton RW, Baildam EM, Turner AJ, Arkwright PD. Recurrent hemiplegia associated with cerebral vasculopathy following third trimester maternal herpes zoster infection. Dev Med Child Neurol. 2006;48(12):991-3. https://doi.org/10.1017/ S0012162206002179

4. Nagel MA, Traktinskiy I, Azarkh Y, KleinschmidtDeMasters B, Hedley-Whyte T, Russman A, et al. Varicella zoster virus vasculopathy: Analysis of virus infected arteries. Neurology. 2011 Jul;77(4):364-70. https://doi.org/10.1212/WNL.0b013e3182267bfa

5. Gilden DH, Lipton HL, Wolf JS, Akenbrandt W, Smith JE, Mahalingam R, et al. Two patients with unusual forms of varicella-zoster virus vasculopathy.
N Engl J Med. 2002 Nov;347(19):1500-3. https://doi. org/10.1056/NEJMoa020841

6. Horten B, Price RW, Jimenez D. Multifocal varicellazoster virus leukoencephalitis temporally remote from herpes zoster. Ann Neurol. 1981 Mar;9(3):25166. https://doi.org/10.1002/ana.410090308

7. Liberman AL, Nagel MA, Hurley MC, Caprio FZ, Bernstein RA, Gilden D. Rapid development of 9 cerebral aneurysms in varicella-zoster virus vasculopathy. Neurology. 2014;82:2139-41. https:// doi.org/10.1212/WNL.0000000000000503

8. Jain R, Deveikis J, Hickenbottom S, Mukherji SK. Varicella-zoster vasculitis presenting with intracranial hemorrhage. AJNR Am J Neuroradiol. 2003;24:971-4.

9. Shah J, Poonawala H, Keay SK, Serulle Y, Steven A, Gandhi D, et al. Varicella-Zoster Virus vasculopathy: A case report demonstrating vasculitis using blackblood MRI. J Neurol Neurophysiol. 2015;6(6). https://doi.org/10.4172/2155-9562.1000342

10. Takeshita J, Nomura E, Takemaru M, Himeno T, Shimoe Y, Kuriyama M. Rapidly deteriorated lobar intracerebral hemorrhages: Possible association of Varicella Zoster Virus-Vasculopathy. Rinsho Shinkeigaku. 2018;58(4):245-8. https://doi. org/10.5692/clinicalneurol.cn-001144

11. Matsuo K, Uozumi Y, Miyamoto H, Tatsumi S, Kohmura E. Varicella-zoster vasculitis presenting with cerebellar hemorrhage. J Stroke Cerebrovasc Dis. 2015;24(6):e153e155. https://doi.org/10.1016/j. jstrokecerebrovasdis.2015.03.00

12. Nagel MA, Cohrs RJ, Mahalingam R, Wellish MC, Forghani B, Schiller A, et al. The varicella zoster virus vasculopathies: Clinical, CSF, imaging, and virologic features. Neurology. 2008 Mar;70(11):853-60. https://doi.org/10.1212/01.wnl.0000304747.38502. e8

13. Nagel MA, Bubak AN. Varicella Zoster Virus vasculopathy. $J$ Infect Dis. 2018;218(suppl 2):S107,S112. https://doi.org/10.1093/infdis/jiy425

14. Osiro S, Salomon N. Varicella-zoster virus (VZV) multifocal vasculopathy in a patient with systemic lupus erythematosus: A diagnostic and treatment dilemma. IDCases. 2017;8:81 -3. https://doi. org/10.1016/j.idcr.2017.04.010 